\title{
Gamma Discriminating Scintillation Screens for Digital Transfer Method Neutron Imaging
}

\author{
Aaron Craft ${ }^{1, a^{*}}$, Christian Grünzweig ${ }^{2, b}$, Manuel Morgano ${ }^{2, c}$ William Chuirazzi $^{1, d}$, \\ and Eberhard Lehmann ${ }^{2, \mathrm{e}}$ \\ ${ }^{1}$ Idaho National Laboratory, PO Box 1625, MS 2211, Idaho Falls, ID 83415, USA \\ ${ }^{2}$ Paul Scherrer Institute, Laboratory for Neutron Scattering and Imaging, CH-5232 Villigen PSI, \\ Switzerland \\ aaaron.craft@inl.gov, bchristian.gruenzweig@psi.ch, 'manuel.morgano@psi.ch, \\ dwilliam.chuirazzi@inl.gov, eeberhard.lehmann@psi.ch
}

Keywords: Neutron Imaging, Scintillator Screen, Indirect Transfer Method, Digital Imaging, Post Irradiation Examination, Nondestructive Evaluation

\begin{abstract}
A collaborative project between Idaho National Laboratory (INL) and Paul-Scherrer Institute (PSI) is investigating a new type of scintillation screen that uses ZnS scintillator material with a dysprosium neutron converter instead of traditional prompt converters such as ${ }^{6} \mathrm{Li}$. Such a screen exposed to a neutron beam creates a latent image by neutron activation of the dysprosium in the scintillator screen. The activated screen is transported into a camera box allowing the camera to read a digital image from the photons emitted by the activated scintillation screen. Such an imaging system combines modern camera-based system architecture with the approach of traditional indirect transfer method radiography. The results show for the first time that the combination of dysprosium with a scintillation material like ZnS can produce light which is measurable under common camera-based detection conditions and that neutron radiographic images of reasonable quality can be produced. The resolution was poorer than expected at $\sim 300 \mu \mathrm{m}$, but is on the order of the desired resolution of $100 \mu \mathrm{m}$. Potential improvements and additional converter materials may be investigated in the future that could increase the light output and improve spatial resolution.
\end{abstract}

\section{Introduction}

As the demand for power increases worldwide, an emphasis has been placed on nuclear energy to meet this need. New nuclear reactor designs utilize a variety of fuels, which must be properly studied and characterized before they can be commercially manufactured. Neutron radiography provides more comprehensive information about the internal geometry of irradiated nuclear fuels and components than any other nondestructive examination technique to date [1]. Transfer method neutron radiography can be used to study irradiated materials despite the very high gamma radiation fields emitted from them [2,3,4]. In the transfer method, a cassette of converter foils is placed in a neutron beam behind an object and exposed to the neutron beam. The converter foils become activated in the pattern of the neutron beam, forming a latent image as a pattern of activation on the foil. The activated foils are coupled to an imaging medium (e.g. film [2], image plates [3]), which are exposed by the decay radiation as the foil decays. Another technique is to use image plates containing dysprosium [4]. The main advantage of this method is its complete insensitivity to gamma rays. However, it is very time consuming and labor intensive.

Current neutron radiography work is trending towards the use of digital systems due to their higher detection efficiency, position stability, flexibility in the field-of-view, high spatial 
resolution, and faster image acquisition. Most digital systems rely on ${ }^{6} \mathrm{LiF} / \mathrm{ZnS}: X$ scintillator screens placed directly in the neutron beam to produce an image. These screens are sensitive to the gamma rays, which has precluded use of camera-based imaging systems for examination of irradiated materials because the object itself is a strong source of gamma radiation.

This paper discusses a project to develop a new type of scintillator screen that combines some of the benefits of modern digital imaging systems with the indirect transfer method by replacing the promptly-decaying neutron converter material with dysprosium which decays over time. Different configurations of scintillator material and dysprosium converter were fabricated. These novel screens were tested to determine their light output during exposure and subsequent decay, and to measure the effective spatial resolution.

\section{Proposed Imaging Methodology for Using Dysprosium-Based Screens}

The proposed imaging process for dysprosium-based scintillator screens is a hybrid between the indirect transfer method and the common direct imaging method with camera-based systems. An illustration of the proposed process can be found in Figure 1. The scintillator screen is first placed behind the imaging object in the neutron beam and exposed directly in the neutron beam. The scintillator screen becomes activated in the neutron beam, creating a latent image in the pattern of activated dysprosium on the scintillator screen. This first step is analogous to the indirect transfer method. After some time, either the neutron beam is turned off (shutter closed) or the activated scintillator screen is transported out of the beam into a light-tight camera box, the latter of which is depicted in Figure 1. The decay products from the activated dysprosium interact with the scintillator material, emitting visible light that is read by the digital camera. In the present tests, the beam shutter was closed to turn off the neutron beam instead of physically moving the screens.
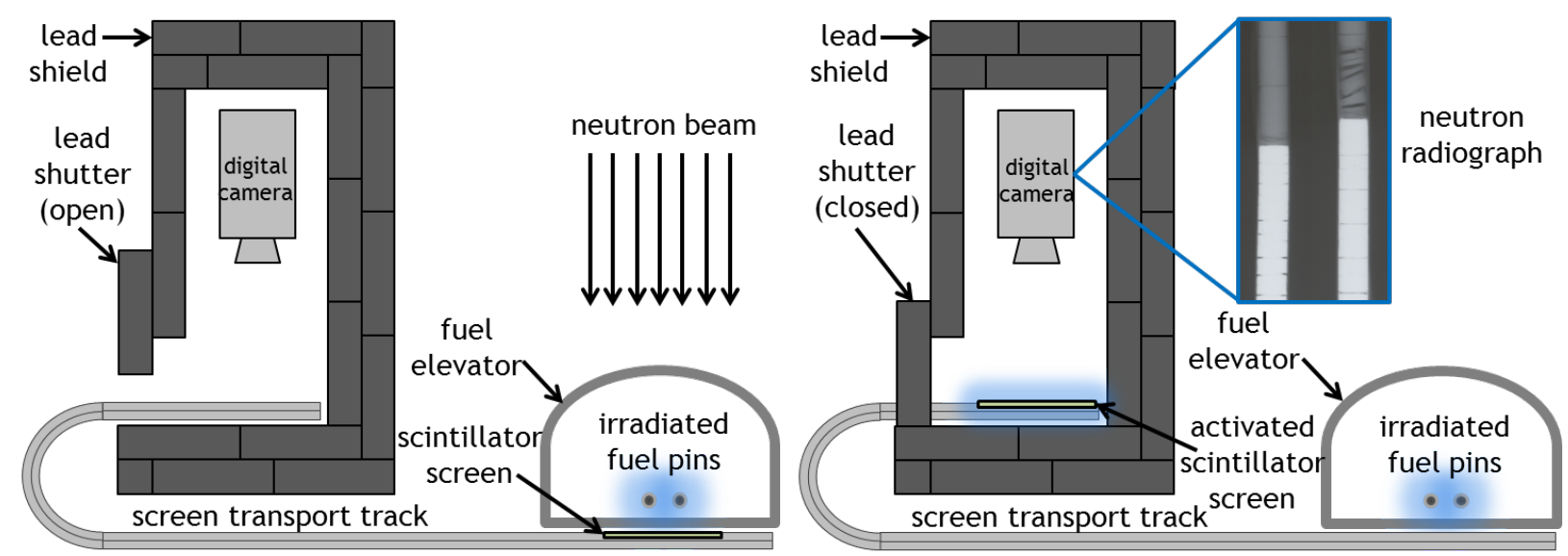

Figure 1. Illustration of the digitial indirect neutron imaging with scintillator screen concept. (Left) The screen is placed in the beamline and exposed behind the imaging object. (Right) The screen is then transported away from the fuel into the camera box and the digital camera captures the latent image on the scintillator screen.

\section{Scintillator Screens}

Scintillator screens were manufactured with a variety of compositions and thicknesses. The $\mathrm{ZnS}: \mathrm{Cu}$ scintillator was mixed with $\mathrm{Dy}_{2} \mathrm{O}_{3}$ for some screens and was deposited directly onto a dysprosium foil for others. Natural dysprosium consists of seven stable isotopes, but ${ }^{164} \mathrm{Dy}$, which comprises $28.18 \%$ of natural dysprosium, is the isotope useful in imaging. When ${ }^{164} \mathrm{Dy}$ absorbs a neutron, it can decay in one of two paths, shown in Equations 1 and 2. Dysprosium 
was chosen because its shorter decay path has a quick half-life (1.26 minutes) and large absorption cross-section ( $\sim 000 \mathrm{~b}$ ), making it suitable for quickly producing neutron radiographs [5]. This is especially useful in an application when many shots must be taken, such as computed tomography.

$$
\begin{aligned}
& { }_{66}^{164} \mathrm{Dy}+n \stackrel{\sigma_{a} \approx 1700 \mathrm{~b}}{\longrightarrow}{ }_{66}^{165} \mathrm{Dy} \stackrel{t_{1 / 2}=2.33 \mathrm{~h}}{\longrightarrow} \beta^{-}+{ }_{67}^{165} \mathrm{Ho} \\
& { }_{66}^{164} \mathrm{Dy}+n \stackrel{\sigma_{a} \approx 1000 \mathrm{~b}}{\longrightarrow}{ }_{65 \mathrm{~m}} \mathrm{Dy} \stackrel{t_{1 / 2}=1.26 \mathrm{~m}}{\longrightarrow} I T_{\gamma}+{ }_{66}^{165} \mathrm{Dy}
\end{aligned}
$$

A total of 23 screens, each 25 $\mathrm{mm} \times 25 \mathrm{~mm}$ square, were fabricated with varying substrate, converter material, phosphor grain size, material ratio, and scintillator thickness. A list of all 23 screens and their properties is detailed in Table 1. Regarding the $\mathrm{ZnS}: \mathrm{Cu}$ grain size, three sizes were used: fine $(\mathrm{d} 50=3.05 \mu \mathrm{m})$, medium $(\mathrm{d} 50=4.67 \mu \mathrm{m})$ and large $(\mathrm{d} 50=9.67 \mu \mathrm{m})$.

\section{Measurements}

The scintillator screens were tested at the NEUTRA beamline at Paul Scherrer Institute (PSI) [6]. The light output of each screen was measured with individual images taken every 2 seconds for total exposure times of 1-20 minutes. Dark-field and open-beam images were acquired and used to correct the images per standard procedures. Light output was analyzed for both the exposure and decay phases of the experiments.

Since the light output of a scintillator is proportional to its activity, light production increases while the screen is exposed to the neutron beam, then decays once the neutron exposure ceases. The screens were exposed for periods of 1,5 and 10 minutes then allowed to decay with the beam turned off. Images were acquired read every 2 seconds. The light output over time was then integrated to determine the total light output during the 5-minute decay phase. The results of the $25 \mathrm{~mm} \times 25 \mathrm{~mm}$ screens are shown in Figure 2. Experimental results revealed that samples \#3 and \#4, both consisting of an absorber material of $\mathrm{Dy}_{2} \mathrm{O}_{3}$ in a 1:2 wt. ratio with large grain $\mathrm{ZnS}: \mathrm{Cu}$, produced the greatest light output for the screens on an aluminum substrate. Some improvement in light output was seen for similar screens on a dysprosium foil substrate (i.e. compare \#3 and \#4 to \#13 and \#14). Figure 3 shows the light output per pixel measured every 
two seconds during a 5-minute exposure and subsequent decay phases for the seven screens that exhibited the highest light output (\#'s 3-4, 13-14 \& 20-22).

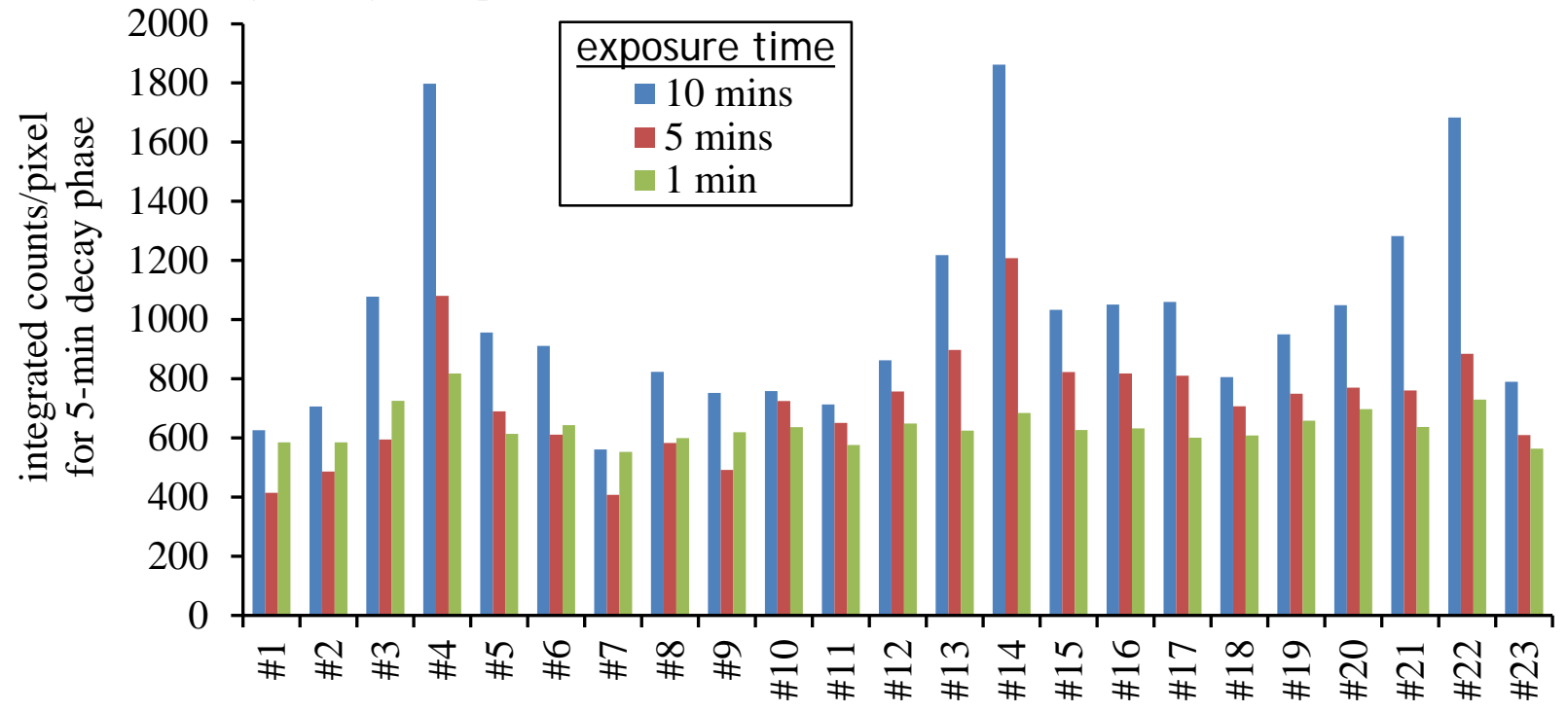

Figure 2. The integrated light output of scintillator screens measured in counts per pixel during the 5-minute decay phase.

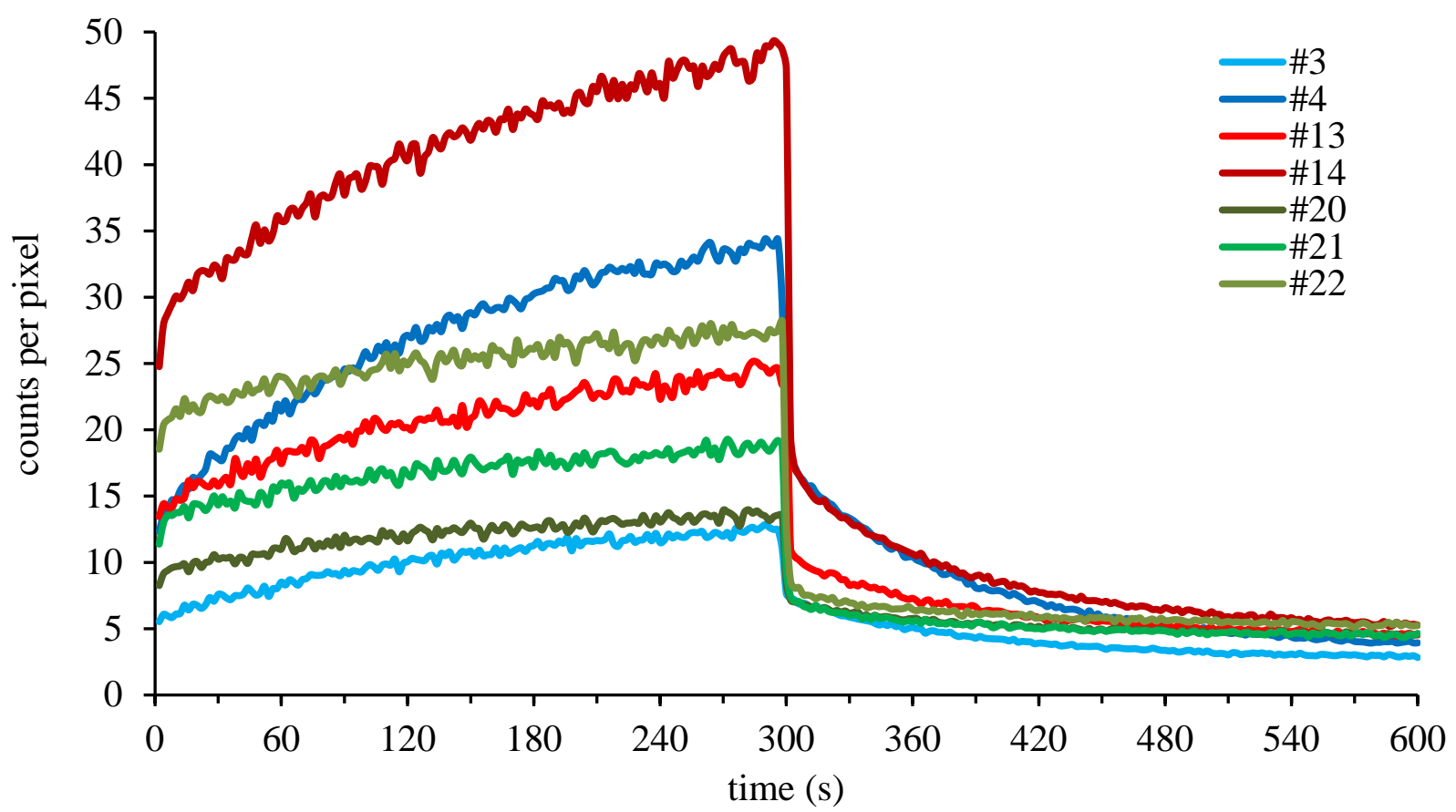

Figure 3. The light output per pixel of the seven screens that exhibited the highest light output. Individual images were taken every 2 s during 5-minute exposure and 5-minute decay phases. The light output builds up as the screens are exposed to the neutron beam for a longer amount of time. The beam is shut off after 300 seconds and the screens' light output decays away with time.

The brightest seven screens were subjected to a 20-minute neutron exposure, and the results are shown in Figure 4. Due to limited beam time, some screens were not allowed to decay completely (takes $\sim 24$ hours for ten half-lives) and residual decay may offset the light output 
values in Figure 4 compared to Figure 3, but the buildup and decay behaviors can still be compared. Recall from Table 1 that screens \#3 and \#4 mix converter with scintillator, \#13 and \#14 have scintillator material deposited on a converter substrate, and screen \#20, \#21 and \#22 include both mixed converter and a converter substrate. As expected, screens with both mixed converter and a converter substrate (screens \#13 and \#14) provided the highest light output. Mixing the scintillator with $\mathrm{Dy}_{2} \mathrm{O}_{3}$ (screens \#3 and \#4) provided higher light output than scintillator deposited on a dysprosium foil (screens \#21 and \#22) for the same scintillator thickness. Thus, mixing converter material with the scintillator was more effective in producing high light output than depositing scintillator on a converter substrate. Also, the difference in light output was much less for the thinner, $100 \mu \mathrm{m}$ thick screens (comparing screens \#3 to \#21, and \#4 to \#22), suggesting that the beta particle from activated dysprosium produces the majority of the light in the first $100 \mu \mathrm{m}$ of scintillator. Furthermore, for screens with scintillator material on a converter substrate, there is only marginal increase in light output by increasing the screen thickness to $200 \mu \mathrm{m}$ (compare screens \#21 and \#22). The screens provided sufficient integrated light output to produce usable images, though there is much room for improvement to increase the light output.
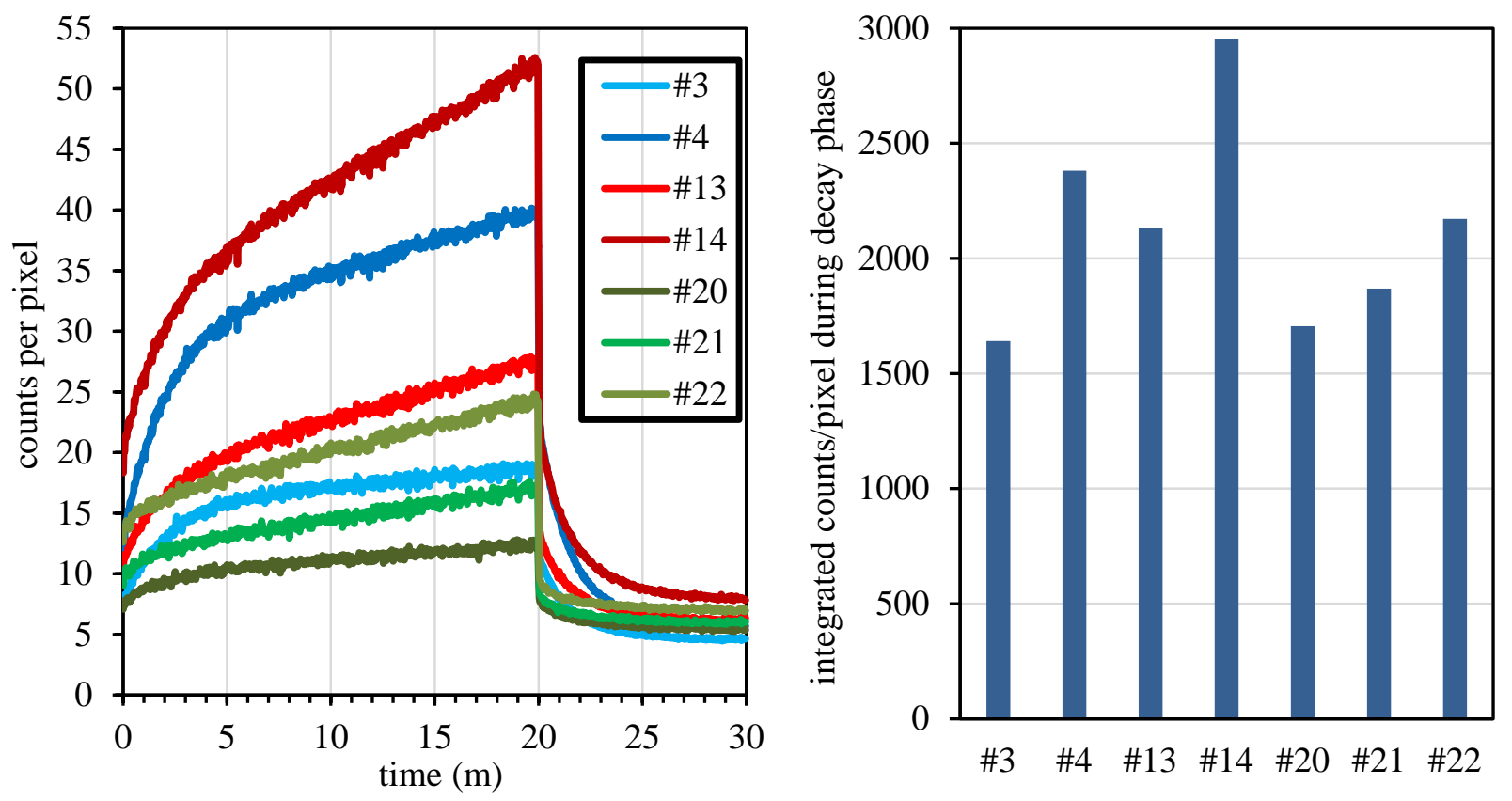

Figure 4. (Left) Light output measured every 2 seconds during the 20-minute exposure and 10minute decay. (Right) Integrated light output for the 10-minute decay phase.

In addition to light output, the spatial resolution of each scintillator was tested. This was accomplished by imaging a Siemens star resolution test pattern with each scintillator screen. The exposure time for the resolution testing was 20 minutes. Upon obtaining the images, the images were gamma-spot filtered, dark-field corrected, and open-beam normalized. Multiple dark-field and open-beam images were acquired, and the median values used for corrections. The test patterns were then analyzed to see the smallest discernable line-pairs. Figure 5 shows images taken with the seven brightest scintillator screens. The resolution was measured by taking the tangential line profile of a circle centered on the Siemens star and extending the radius out to the location where the star's spokes first become visible. A standard $200 \mu \mathrm{m}$ thick ${ }^{6} \mathrm{LiF} / \mathrm{ZnS}$ screen 
was used as a comparison. The best spatial resolution from the screens in this experiment was $\sim 300 \mu \mathrm{m}$, which was not as good as the $\sim 200 \mu \mathrm{m}$ given by the standard screen.

\section{Conclusions}

The purpose of this study was to develop and test a dysprosium-based scintillator screen that could be used in a novel, hybrid neutron imaging method combining the indirect transfer method with digital camera-based neutron radiography. The results show for the first time that dysprosium can be used with a standard scintillator phosphor to create a scintillator screen that can produce a useful amount of light after exposure to a neutron beam. Although the resolution was not as high as desired, the resulting radiographs were of reasonable quality. With further improvements (e.g. using enriched ${ }^{164}$ Dy), dysprosium-based screens could be usable for imaging highly-radioactive objects. Additionally, other converter materials may be investigated in future work with the goal of improving light

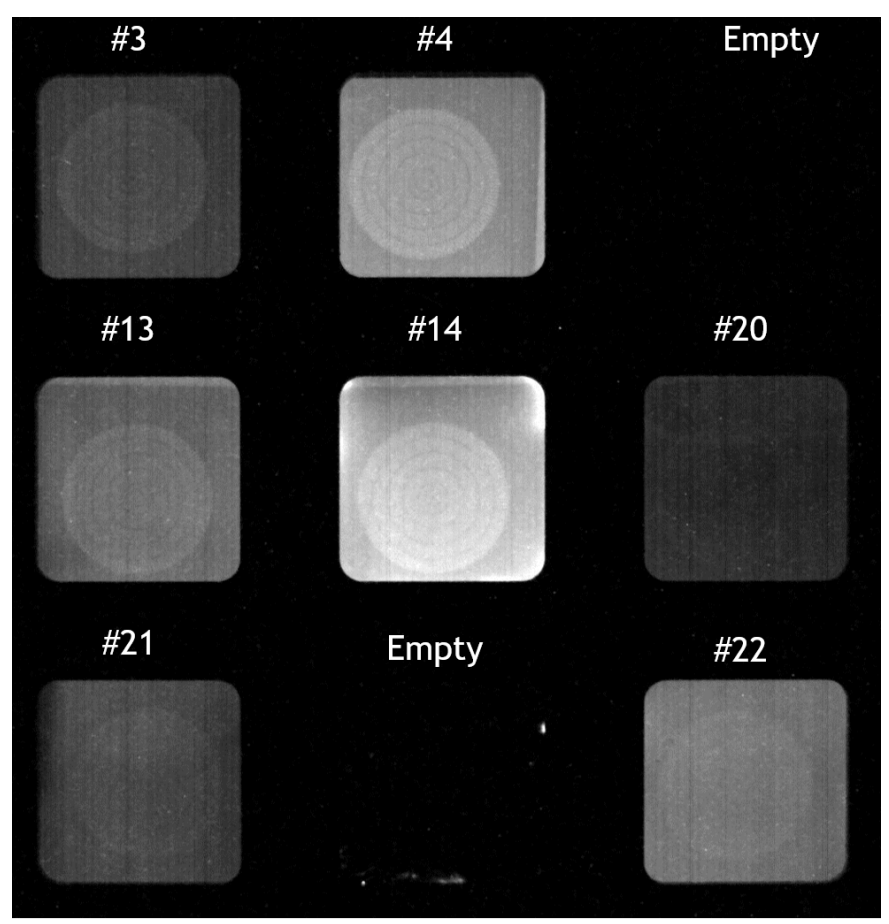

Figure 5. Radiographs of a Seimens star acquired with the brightest seven scintillator screens. output and spatial resolution.

\section{References}

[1] A.E. Craft and J.D. Barton, "Applications of neutron radiography for the nuclear power industry,” Physics Procedia 88 (2017) 73-80. https://doi.org/10.1016/j.phpro.2017.06.009

[2] A.E. Craft, D.M. Wachs, M.A. Okuniewski, D.L. Chichester, W.J. Williams, G.C. Papaioannou, \& A.T. Smolinski, "Neutron radiography of irradiated nuclear fuel at Idaho National Laboratory." Physics Procedia, 69 (2015) 483-490. https://doi.org/10.1016/j.phpro.2015.07.068

[3] A.E. Craft, G.C. Papaioannou, D.L. Chichester, \& W.J. Williams, "Conversion from film to image plates for transfer method neutron radiography of nuclear fuel." Physics Procedia, 88 (2017) 81-88. https://doi.org/10.1016/j.phpro.2017.06.010

[4] P. Vontobel, M. Tamaki, N. Mori, T. Ashida, L. Zanini, E.H. Lehmann, \& M. Jaggi, "Postirradiation analysis of SINQ target rods by thermal neutron radiography." Journal of nuclear materials, 356(1-3) (2006) 162-167. https://doi.org/10.1016/j.jnucmat.2006.05.033

[5] E.M. Baum, H.D. Knox, and T.R. Miller, "Chart of the Nuclides,” 16th ed., Knolls Atomic Power Laboratory (2002).

[6] E.H. Lehmann, P. Vontobel, and L. Wiezel, "Properties of the Radiography Facility NEUTRA at SINQ and its Potential for Use as European Reference Facility.” Nondestructive Testing \& Eval 16 (2001) 191-202. https://doi.org/10.1080/10589750108953075 